\title{
Mathematical Model of VOCs Emission in Three-layer Building Materials
}

\author{
Du Zhehua ${ }^{1, *}, \operatorname{Lin} \mathrm{Xin}^{2}$ \\ ${ }^{1}$ Wuhan Second Ship Design and Research Institute, 430205 Wuhan, China \\ ${ }^{2}$ Hubei Province Engineering Consulting Co., LTD., 430071 Wuhan, China
}

\begin{abstract}
A simple mathematical model is proposed to account for emissions of Volatile Organic Compounds (VOCs) from three-layer building materials. The model considers both the diffusion within three layer building materials and the mass transfer resistance through the air boundary layer. A general solution method based on Laplace transform is presented. Compared to other models capable of accounting for emissions of VOCs from multi layer building materials, the present model is fully analytical instead of being numerical. The present model was validated by the experimental data from the specially designed test. The results indicated that there was a good agreement between the model predictions and the experimental data. It can also be seen from calculation that model ignoring the boundary layer resistance cannot fully reflect the real situation.
\end{abstract}

\section{Introduction}

Volatile Organic Compounds (VOCs) emitted by building materials such as floor coverings and wood products are considered to be one of the main threats to human health. Therefore, it is necessary to have a deep understanding of VOCs emission characteristics and their propagation mechanism in building materials and indoor. In order to accurately describe the emission characteristics of VOCs, several mathematical models have been proposed. Little et al. (1994) pioneered the emission model of VOCs in single-layer dry materials. However, since this model ignores mass transfer resistance in the air, the concentration of VOCs in the air at the initial stage of emission is overestimated [1]. Shin et al. (2003) used Little's model to simulate the divergence process in the carpet and obtained the model parameters through corresponding experimental data [2]. Yang et al. (2001) developed a numerical model to simulate the divergence process of VOCs from a single layer of dry material, and conducted an experimental study [3]. Zhao et al. (2002) developed an analytical model that can study instantaneous pollution sources based on the ideas of Little et al. (1994) [4]. Huang and Haghighat (2002) developed a numerical model that introduced a gas-phase mass transfer coefficient to describe the mass transfer resistance [5], which can be solved by the finite difference technique. Xu and Zhang (2003) proposed an improved mass transfer model, including the analytical solution of one-dimensional diffusion equation in the model proposed by Huang and Hagighat (2002) [6]. However, the model proposed by $\mathrm{Xu}$ and Zhang (2003) is not a complete analytical solution. Because it is related to the concentration of
VOCs in the air, which is an unknown function of time. Therefore, the concentration in the material and the mass balance equation in the air must be solved simultaneously by finite difference technology. That is to say, this model still belongs to the category of numerical solution, and it is not convenient to compare this model with Little's fully analytical solution model. Deng and Kim (2004) obtained the analytical solution of Huang and Haghighat (2002) model through Laplace transform [7].

The above models can only be used to predict the divergence process in a single-layer building material with uniform physical properties. However, many composite building materials in reality are multi-layer materials composed of several different substances. Each layer may have a different VOCs diffusion coefficient, and their material-air distribution coefficient may also be different. The inner layer may be the "source" or "sink" of VOCs to the outer layer, which may affect the concentration of VOCs in the air. Therefore, it is necessary to develop a model that can describe the phenomenon of VOCs emanating from multilayer materials. Kumar and Little (2003) proposed a two-layer material VOCs divergence model [8]. The concentration distribution of VOCs in the double-layer material and the concentration of VOCs in the air mainstream are obtained, but mass transfer resistance in the air boundary layer is ignored. Zhang and Niu (2004) studied rooms using multi-storey materials. The model proposed by them takes into account mass transfer resistance in the air boundary layer, and they use the finite difference method to solve the governing equation [9]. However, finite difference method is too time-consuming to solve, which brings inconvenience to the application.

*Corresponding author: shunli878@163.com 
This paper presents a simple mathematical model which can easily predict the dispersion of VOCs in three-layers building materials. This model considers the mass transfer resistance inside the material and the air boundary layer. The general solution of VOCs concentration in air is obtained by Laplace transform.

\section{Model export}

The interior has three layers of structural building materials : $\left(x_{1}=0, x_{2}\right),\left(x_{2}, x_{3}\right),\left(x_{3}, x_{4}\right)$. To simplify the problem, the following assumptions are made:

- The authors, in the form: initials of the first names followed by last name (only the first letter capitalized with full stops after the initials),

- Each layer of building material has uniform physical properties and the same initial VOCs concentration.

- During emission process, vapor pressure of VOCs maintains a thermal balance at the interface between the material and the air.

- Although mass transfer may be caused by the coupled effects of temperature gradient, pressure gradient, external force and concentration gradient, only the concentration gradient of VOCs is used as the driving force for mass transfer in this analysis.

- No chemical reaction occurs when VOCs is generated or consumed inside building materials.

- VOCs diffusion in each layer is a one-dimensional problem.

Based on the above assumptions, the governing equations of mass transfer and equilibrium of VOCs in three-layer material are given.

The instantaneous governing equation of VOCs diffusion in each layer of material is:

$$
\frac{\partial C_{m}}{\partial \tau}=D_{m} \frac{\partial^{2} C_{m}}{\partial x^{2}}(m=1,2,3)
$$

$C_{m}$ is the concentration of VOCs in the m-th layer of material. $D_{m}$ is the diffusion coefficient in the m-th layer of material. $\tau$ is time. $x$ is the diffusion direction of VOCs in the material; $i=1$ indicates the bottom layer of the material, $i=3$ represents the top layer of material adjacent to air. The initial conditions for each layer of equation are as follows.

$$
C_{m}(\tau=0)=C_{m, 0}
$$

$C_{m, 0}$ is the initial concentration of VOCs in the m-th layer of material.

Since the diffusion between adjacent layers of materials is constrained by the conservation of mass, the mass diffusion flux at the interface of two adjacent layers is continuous, so the following formula can be derived.

$$
\left.q\right|_{x_{m+1}}=-\left.D_{m} \frac{\partial C_{m}}{\partial x}\right|_{x_{m+1}}=-\left.D_{m+1} \frac{\partial C_{m+1}}{\partial x}\right|_{x_{m+1}}
$$

$q$ is the mass diffusion flux of the two adjacent layers. Because the diffusion coefficient of each layer is different, the concentration of VOCs is discontinuous, so according to Henry's law, there is following formula.

$$
\left.\frac{C_{m}}{K_{m, a}}\right|_{x_{m+1}}=\left.\frac{C_{m+1}}{K_{m+1, a}}\right|_{x_{m+1}}
$$

$K_{m, a}$ and $K_{m+1, a}$ are the material-air distribution coefficient of the $m$ layer and $(m+1)$ layer, respectively.

Equation (4) shows that VOCs concentration on both sides of the material's adjacent interlayer interface is discontinuous. The introduction of gas phase equilibrium concentration $C_{e}=C_{m} / K_{m, a}$ can keep the concentration of VOCs continuous on the interface between different layers. Equations $(1) \sim(4)$ can be rewritten as:

$$
\begin{aligned}
& \frac{\partial C_{e}}{\partial \tau}=D_{m} \frac{\partial^{2} C_{e}}{\partial x^{2}}(m=1,2,3) \\
& C_{e}(\tau=0)=C_{m, 0} / K_{m, a} \\
& \left.q\right|_{x_{m+1}}=-\left.D_{m} K_{m, a} \frac{\partial C_{e}}{\partial x}\right|_{x_{m+1}}=-\left.D_{m+1} K_{m+1, a} \frac{\partial C_{e}}{\partial x}\right|_{x_{m+1}} \\
& \left.C_{e}\right|_{x_{m+1^{+}}}=\left.C_{e}\right|_{x_{m+1}}
\end{aligned}
$$

Assuming that three layers of building material are placed on the stainless steel floor of the room, there is no mass flux at its lower boundary.

$$
\begin{aligned}
& \left.\frac{\partial C_{e}}{\partial x}\right|_{x_{1}}=0 \\
& \left.\bar{q}\right|_{x_{1}}=0
\end{aligned}
$$

The material - air interface is the third type of mass transfer boundary condition, which is constrained by the following relation.

$$
\left.q\right|_{x_{4}}=h\left(\left.C_{e}\right|_{x_{4}}-C_{a}\right)
$$

$h$ is gas-phase mass transfer coefficient. $C_{a}$ is the concentration of VOCs in the air. An equation for $C_{a}$ can be obtained from the conservation of mass of VOCs in the room. Since the inlet VOCs concentration is zero, the mass conservation equation can be rewritten as the following formula.

$$
\frac{\partial C_{a}}{\partial t}=\left.L q\right|_{x_{4}}-N C_{a}
$$

$L$ is load ratio and $N$ is room air exchange rate.

Generally speaking, the finite difference method can be used to solve the equations (5) - (12), but the application is inconvenient because solution process is too time-consuming. In this study, Laplace transformation is used to solve the problem.

According to the method of Carslaw and Jaeger (1986) [10], considering the initial concentration field in the material, the Laplace conversion of the m-th layer is as follows.

$$
\begin{aligned}
& \left(\left.\left.\lambda \overline{C_{e}}\right|_{x_{m+1}} \lambda \bar{q}\right|_{x_{m+1}}\right)^{T}=H_{m}\left(\left.\left.\lambda \overline{C_{e}}\right|_{x_{m}} \lambda \bar{q}\right|_{x_{m}}\right)^{T}+G_{m}(13) \\
& G_{m}=\left(C_{m, 0}\left(1-A_{m}\right) / K_{m, a}-E_{m} C_{m, 0} / K_{m, a}\right)^{T}(14) \\
& H_{m}=\left(\begin{array}{ll}
A_{m} & B_{m} \\
E_{m} & F_{m}
\end{array}\right) \\
& p_{m}=(\lambda / D)^{0.5} \\
& A_{m}=\cosh p_{m} \delta_{m}
\end{aligned}
$$




$$
\begin{aligned}
& B_{m}=-\frac{\sinh p_{m} \delta_{m}}{D_{m} K_{m, a} p_{m}} \\
& E_{m}=-D_{m} K_{m, a} p_{m} \sinh p_{m} \delta_{m} \\
& F_{m}=\sinh p_{m} \delta_{m} \\
& A_{m} F_{m}-B_{m} E_{m}=1
\end{aligned}
$$

$\lambda$ is Laplace variable. $\left.\overline{C_{e}}\right|_{x_{m+1}}$ and $\left.\bar{q}\right|_{x_{m+1}}$ are

Laplace transform of VOCs concentration and diffusion flux in the $(\mathrm{m}+1)$ th layer, respectively. $\delta_{m}=x_{m+1}-x_{m}$ is the thickness of the $\mathrm{m}$-th layer of material. Applying equation (13) to the third layer and performing matrix operations, the following matrix form relational expressions in the third layer material adjacent to air can be obtained.

$$
\begin{aligned}
& \left(\left.\left.\lambda \overline{C_{e}}\right|_{x_{4}} \lambda \bar{q}\right|_{x_{4}}\right)^{T}=\left(\begin{array}{ll}
A & B \\
E & F
\end{array}\right)\left(\left.\left.\lambda \overline{C_{e}}\right|_{x_{1}} \lambda \bar{q}\right|_{x_{1}}\right)^{T}+\left(\begin{array}{c}
G_{c} \\
G_{q}
\end{array}\right) \\
& \left(\begin{array}{ll}
A & B \\
E & F
\end{array}\right)=H_{3} H_{2} H_{1} \\
& \left(\begin{array}{l}
G_{c} \\
G_{q}
\end{array}\right)=G_{3}+H_{3} G_{2}+H_{3} H_{2} H_{1}
\end{aligned}
$$

The following equation can be obtained by combining equations (10) and (19).

$$
\left.\lambda E \overline{C_{e}}\right|_{x_{4}}=\left.\lambda \overline{A q}\right|_{x_{4}}+E G_{c}-A G_{q}
$$

By using Laplace transform, equations (11) and (12) can be written as follows.

$$
\begin{aligned}
& \left.\bar{q}\right|_{x_{4}}=\left.h \overline{C_{e}}\right|_{x_{4}}-h \overline{C_{a}} \\
& \lambda \overline{C_{a}}=\left.L \bar{q}\right|_{x_{4}}-N \overline{C_{a}}
\end{aligned}
$$

From equations (25), (26) and (27), the following equations can be obtained.

$$
\begin{aligned}
& \overline{C_{a}}=\frac{L\left(E G_{c}-A G_{q}\right)}{\lambda\left(\left(\frac{\lambda+N}{h}+L\right) E-(\lambda+N) A\right)} \\
& \left.\bar{q}\right|_{x_{4}}=\frac{(\lambda+N)\left(E G_{c}-A G_{q}\right)}{\lambda\left(\left(\frac{\lambda+N}{h}+L\right) E-(\lambda+N) A\right)}
\end{aligned}
$$

The solution of equations (28) and (29) can be obtained using the inversion theorem.

$$
\begin{aligned}
& C_{a}=L \sum_{k=1}^{\infty} X_{k} e^{-r_{k}^{2} \delta_{1}^{-2} D_{1} t} \\
& q_{x 4}=\sum_{k=1}^{\infty}\left(N-r_{k}^{2} \delta_{1}^{-2} D_{1}\right) X_{k} e^{-r_{k}^{2} \delta_{1}^{-2} D_{1} t} \\
& X_{k}= \\
& \left.\frac{E G_{c}-A G_{q}}{\lambda\left((E / h-A)+((\lambda+N) / h+L) E^{\prime}-(\lambda+N) A^{\prime}\right)}\right|_{\lambda=-r_{k}^{2} \delta_{1}^{2} D_{1}}
\end{aligned}
$$

$$
\left(\begin{array}{ll}
A^{\prime} & B^{\prime} \\
E^{\prime} & F^{\prime}
\end{array}\right)=\frac{d}{d \lambda} H_{3} H_{2} H_{1}
$$

$r_{k}$ is the positive root of this equation.

$$
\begin{aligned}
& \left(\left(N-r_{k}^{2} \delta_{1}^{-2} D_{1}\right) / h+L\right) /\left.E\right|_{\lambda=-r_{k}^{2} \delta_{1}^{-2} D_{1}} \\
& -\left.\left(N-r_{k}^{2} \delta_{1}^{-2} D_{1}\right) A\right|_{\lambda=-r_{k}^{2} \delta_{1}^{-2} D_{1}}=0
\end{aligned}
$$

Through equations (30) - (34), VOCs concentration in the air and VOCs emission at the material-air interface can be easily obtained by means of computer programming. Because these two series of numbers converge very quickly, usually the first 200 terms can be calculated with sufficient accuracy.

The existing model considers four key parameters: gas-phase mass transfer coefficient $h$, material-air distribution coefficient, mass diffusion coefficient in the material $D_{m}$, and the initial VOCs concentration in the material $C_{m, 0}$. The last three items can be determined through experiments. The gas-phase mass transfer coefficient can be obtained by some empirical correlations.

For laminar flow $(\operatorname{Re}<50000)$, there is the following equation (White, 1988) [11].

$$
S h=0.664 S c^{\frac{1}{3}} \operatorname{Re}^{\frac{1}{2}}
$$

For turbulent flow $(R e>50000)$, there is the following equation (White, 1988) [11].

$$
S h=0.037 S c^{\frac{1}{3}} \operatorname{Re}^{\frac{4}{5}}
$$

$S h$ is Sherwood number, $S c$ is Schmidt number, $R e$ is Reynolds number, $v$ is air kinematic viscosity, $l$ is material characteristic length, and $D_{a}$ is mass diffusion coefficient of VOCs in air.

\section{Model verification}

Since the experimental results of VOCs emission in three-layer building materials have not been found in published literature, only the experimental results of onelayer building materials can be used to verify the model proposed in this paper. Yang et al. (2001) conducted VOCs divergence experiment in an experimental chamber with a volume of $0.5 \mathrm{~m} \times 0.4 \mathrm{~m} \times 0.25 \mathrm{~m}$. They studied the distribution of VOCs from two different particleboards (PB1 and PB2) [3]. The geometric dimension of PB1 and PB2 is $0.212 \mathrm{~m} \times 0.212 \mathrm{~m} \times$ $0.0159 \mathrm{~m}$. The concentration of VOCs in the air at different times was measured in the experiment. Since the particleboard in the experiment is a single layer, in order to verify the model proposed in this paper, it can be assumed that the particleboard is composed of three layers of materials with the same physical properties. The initial concentration and mass transfer diffusion coefficient of VOCs in each layer are the same. The material-air distribution coefficients between different layers are also the same, namely $C_{10}=C_{20}=C_{30}, D_{1}=D_{2}$ $=D_{3}, K_{l, a}=K_{2, a}=K_{3, a}$. Meanwhile, it is assumed that the thickness of three layers is $\delta_{1}=0.012, \delta_{2}=0.002$, and $\delta_{3}$ $=0.0019$, respectively. 
Based on the above assumptions, the model proposed in this paper can be used to predict the divergence of VOCs in particleboard experiments. It can be seen that there is a good agreement between the two. As the experimental results of VOCs emission in three-layer building materials have not been reported, it cannot be compared with the model proposed in this paper. Experiments with three layers of materials are proposed to further verify the model. It can be seen that VOCs concentrations at the interface and the air mainstream are different. It also proves that the model ignoring the mass transfer resistance of the boundary layer cannot fully reflect the real situation.

\section{Conclusion}

In this paper, a simple mathematical model is proposed to predict VOCs emission process in three-layer building materials. The model takes into account mass diffusion coefficient in the material and mass transfer resistance of air boundary layer, and a general solution based on the Laplace transform is obtained. The concentration of VOCs in the air can be expressed by two rapidly converging infinite series. Therefore, the use of this model can easily predict the concentration of VOCs emitted from three-layer building materials in the air. The accuracy of model prediction is verified by experimental result in the literature, and the predicted value is basically consistent with the experimental data. It can also be seen from calculation that model ignoring the boundary layer resistance cannot fully reflect the real situation.

\section{Acknowledgments}

This work was supported by the National Key Research and Development Program (No.2017YFC0307800).

\section{References}

1. J.C. Little, A.T. Hodgson, A.J. Gadgil, Atmospheric Environment, 28, 227 (1994)

2. D.M. Shin, C.N. Kim, D.S. Kim, Journal of SAREK, 15, 40 (2003)

3. X. Yang, Q. Chen, J.S. Zhang, Building and Environment, 36, 1099 (2001)

4. D. Zhao, J.C Little, A.T. Hodgson, Indoor Air, 12, 184 (2002)

5. H. Huang, F. Haghighat, Building and Environment, 37, 1349 (2002)

6. Y. Xu, Y. Zhang, Atmospheric Environment, 37, 2497 (2003)

7. B.Q. Deng, C.N. Kim, Atmospheric Environment, 38, 1173 (2004)

8. D. Kumar, J.C. Little, Atmospheric Environment, 37, 5529 (2003)

9. L.Z. Zhang, J.L. Niu, Building and Environment, 39, 523 (2004)
10. H.S. Carslaw, J.C. Jaeger, Conduction of Heat in Solid (2nd edition), Oxford University Press (1986)

11. F.M. White, Heat and mass transfer, AddisonWesley (1988) 